Supporting Information

\title{
Conformal Macroporous Inverse Opals Oxynitride-Based Photoanode for Robust Photoelectrochemical Water Splitting
}

Lei Ran, ${ }^{\dagger}$ Shi Qiu, ${ }^{\dagger}$ Panlong Zhai, ${ }^{\dagger}$ Zhuwei Li, Junfeng Gao, Xiaomeng Zhang, Bo Zhang, Chen Wang, Licheng Sun, and Jungang Hou*

'L.R., S.Q. and P.Z. equally contributed to this work.

*E-mail: J.Hou (jhou@dlut.edu.cn) 


\section{Experiment and characterization.}

Fabrication of PS spheres template. Fluorine-doped tin oxide (FTO) glass $(1 \mathrm{~cm} \times 2 \mathrm{~cm}$ in size) has been cleaned under sonication by sequentially immersing in ethanol, acetone and distilled water, and then treated with $\mathrm{H}_{2} \mathrm{SO}_{4} / \mathrm{H}_{2} \mathrm{O}_{2} / \mathrm{H}_{2} \mathrm{O}(3: 1: 1$, volume ratio) solution. Monodispersed PS (polystyrene) spheres were prepared by the previous report. ${ }^{1,2}$ The well-ordered colloidal crystal templates comprising polystyrene (PS) spheres arranged on FTO substrate by evaporation-induced self-assembly.

Calculation of solar energy conversion efficiency and IPCE tests. The PEC properties of the photoelectrodes were evaluated by a three-electrode system. According to the Nernst equation $\left(E_{\mathrm{RHE}}=E_{\mathrm{Ag} / \mathrm{AgCl}}+0.059 \mathrm{pH}+0.197\right)$, where $E_{\mathrm{RHE}}$ was the potential vs. a reversible hydrogen potential, $E_{\mathrm{Ag} / \mathrm{AgCl}}$ was the potential vs. $\mathrm{Ag} / \mathrm{AgCl}$ electrode, and $\mathrm{pH}$ was the $\mathrm{pH}$ value of electrolyte. The solar energy conversion efficiency $(\eta)$ of the photoanode was calculated by the photocurrent-potential curves under chopped AM 1.5G sunlight using the equation:

$$
\eta=\left[\left(1.23-V_{\text {app }}\right) \times\left(J_{\text {light }}-J_{\text {dark }}\right) / P_{\text {light }}\right] \times 100 \%
$$

where $V_{\text {app }}$ is the applied potential, $J_{\text {dark }}$ and $J_{\text {light }}$ are the photocurrent density in darkness and under AM 1.5G light, and $P_{\text {light }}$ is the power density of AM $\left.1.5 \mathrm{G}(100 \mathrm{~mW} \mathrm{~cm})^{-2}\right)$.

The wavelength dependent the incident photon to current efficiency (IPCE) was calculated according to the following equation:

$$
\operatorname{IPCE}(\%)=\frac{1240 \times J_{\text {light }}}{\lambda \times P_{\text {light }}} \times 100
$$

where $J_{\text {light }}$ is the photocurrent $(\mathrm{mA}), \lambda$ is the wavelength $(\mathrm{nm})$ of incident radiation, and $P_{\text {light }}$ is the measured light power density at that wavelength $(\mathrm{mW})$. The wavelength dependence of IPCE was measured under monochromatic irradiation from a $300 \mathrm{~W}$ Xe lamp equipped with bandpass filters.

Calculation of charge separation evaluation. The photocurrent density arising from PEC performance $\left(\mathrm{J}_{\mathrm{PEC}}\right)$ can be described as following:

$$
J_{P E C}=J_{a b s} \times \eta_{\text {sep }} \times \eta_{\text {trans }}
$$

where $J_{\mathrm{abs}}$ is the photocurrent density when completely converting the absorbed photons into current $\left(J_{\text {abs }}\right)$. Adding $0.2 \mathrm{M} \mathrm{Na}_{2} \mathrm{SO}_{3}$ as the electrolyte can largely suppress the surface recombination of charge carriers without influencing the charge separation in the electrode bulk (i.e., $\eta_{\text {trans }}$ could be regarded as $100 \%$ ). Therefore, $\eta_{\text {sep }}$ can be determined as following:

$\eta_{\text {sep }}=J_{\mathrm{Na}_{2} \mathrm{SO}_{3}} / J_{\text {abs }}$

$\eta_{\text {trans }}=J_{\mathrm{H}_{2} \mathrm{O}} / \mathrm{Na}_{2} \mathrm{SO}_{3}$

where $J_{\mathrm{H}_{2} \mathrm{O}}$ and $\mathrm{JNa}_{2} \mathrm{SO}_{3}$ is the photocurrent density for $\mathrm{H}_{2} \mathrm{O}$ oxidation and $\mathrm{Na}_{2} \mathrm{SO}_{3}$ oxidation, respectively.

First-principle calculations. To investigate the $\mathrm{BCN} / \mathrm{CsTaWO} \mathrm{G}_{-\mathrm{x}} \mathrm{N}_{\mathrm{x}}$ composite, we have used a co-periodic supercell with lattice mismatch is less than $3 \%$. In order to avoid interaction between adjacent cells, the supercell with every lattice dimension larger $14 \AA$ is used. The first-principles calculations for all the system were performed with the Vienna $A b$ initio Simulation Package (VASP). ${ }^{3}$ Generalized Gradient Approximation (GGA) and 
Perdew-Burke-Ernzerhof (PBE) functions describe electronic exchange and correlation. ${ }^{4}$ The ion-electron interaction is described by the projector-enhanced wave (PAW) method. ${ }^{5}$ The energy cutoff for plane wave is up to $400 \mathrm{eV}$. The Brillouin zone is sampled using the Monkhorst-Pack method with a span of $2 \pi \times 0.03 \AA^{-1}$ in our calculation. ${ }^{6}$ The total energies converged tolerance is $1 \times 10^{-6} \mathrm{eV}$, and ions are relaxed until the max force less than 0.02 eV/Å. 
Supporting Figures.
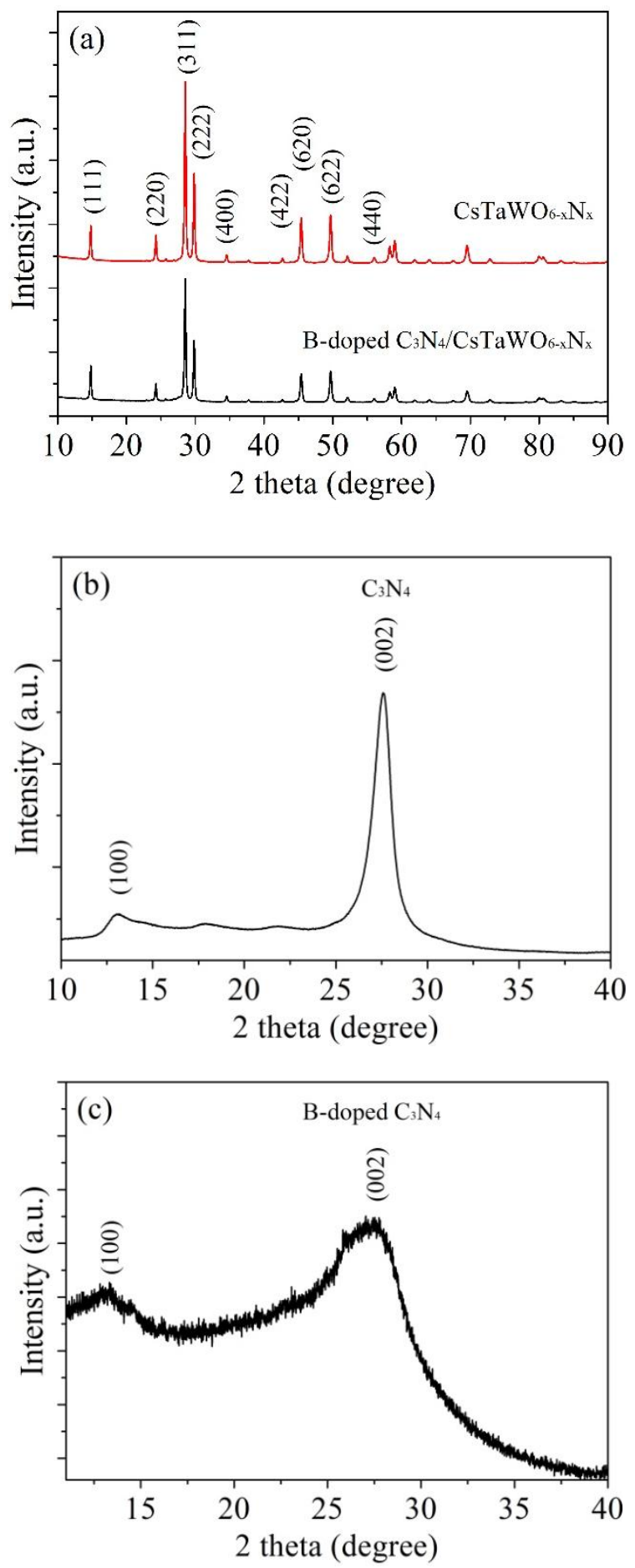

Figure S1. XRD patterns of $\mathrm{C}_{3} \mathrm{~N}_{4}$, B-doped $\mathrm{C}_{3} \mathrm{~N}_{4}, \mathrm{CsTaWO}_{6-\mathrm{x}} \mathrm{N}_{\mathrm{x}}$ and conformal B-doped $\mathrm{C}_{3} \mathrm{~N}_{4} / \mathrm{CsTaWO}_{6-\mathrm{x}} \mathrm{N}_{\mathrm{x}}$ arrays. 

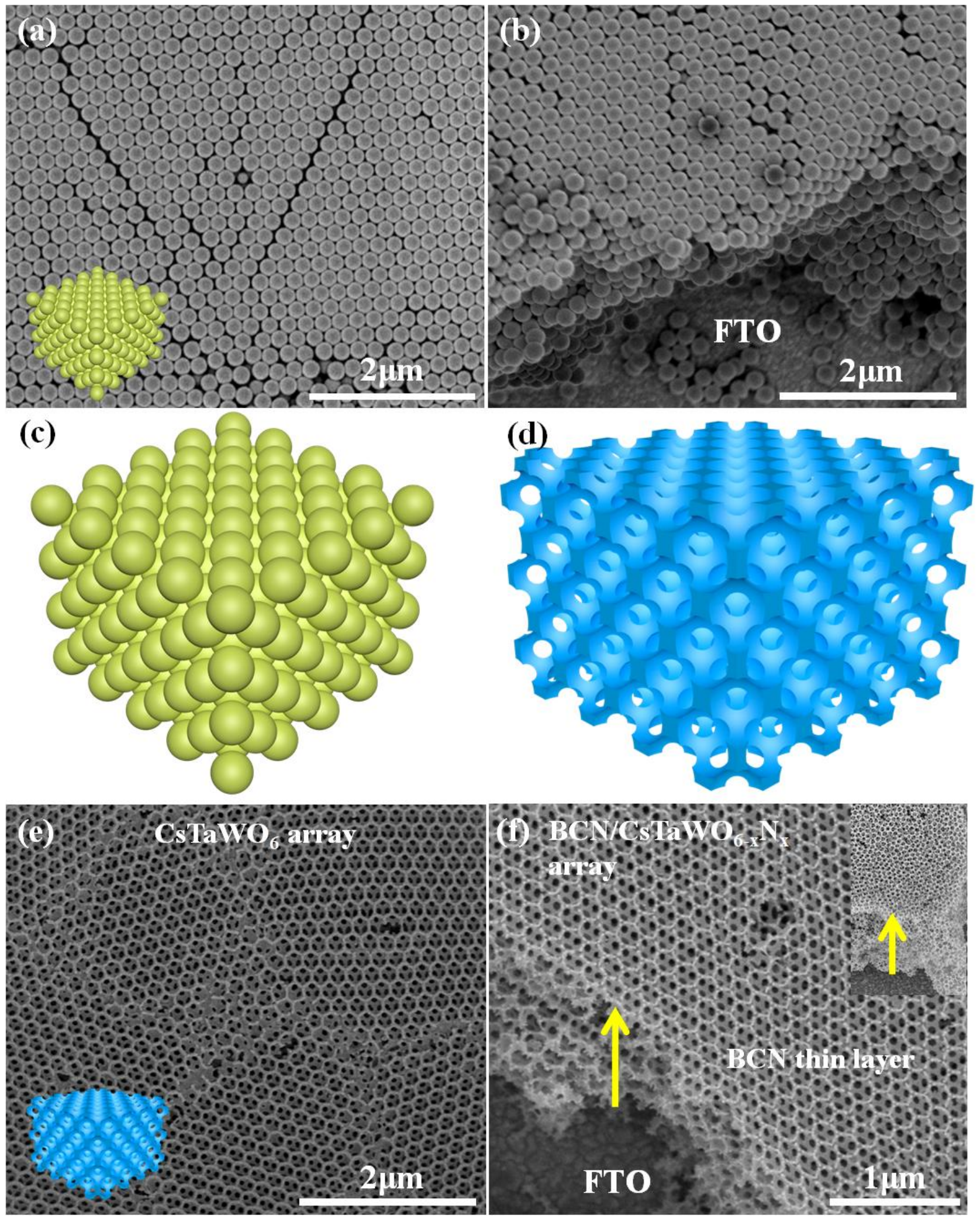

Figure S2. SEM images of (ab) PS spheres arrays, (e) $\mathrm{CsTaWO}_{6}$ inverse opals array and (f) conformal BCN/CsTaWO ${ }_{6-x} \mathrm{~N}_{\mathrm{x}}$ inverse opals array, and (cd) illustrations of PS spheres and inverse opals architecture. 


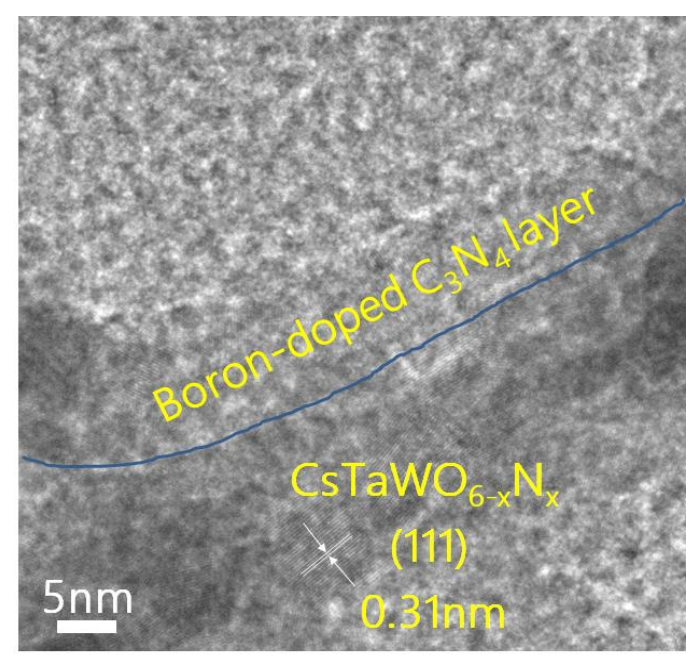

Figure S3. HRTEM image of conformal BCN/CsTaWO ${ }_{6-x} \mathrm{~N}_{x}$ inverse opals architecture.
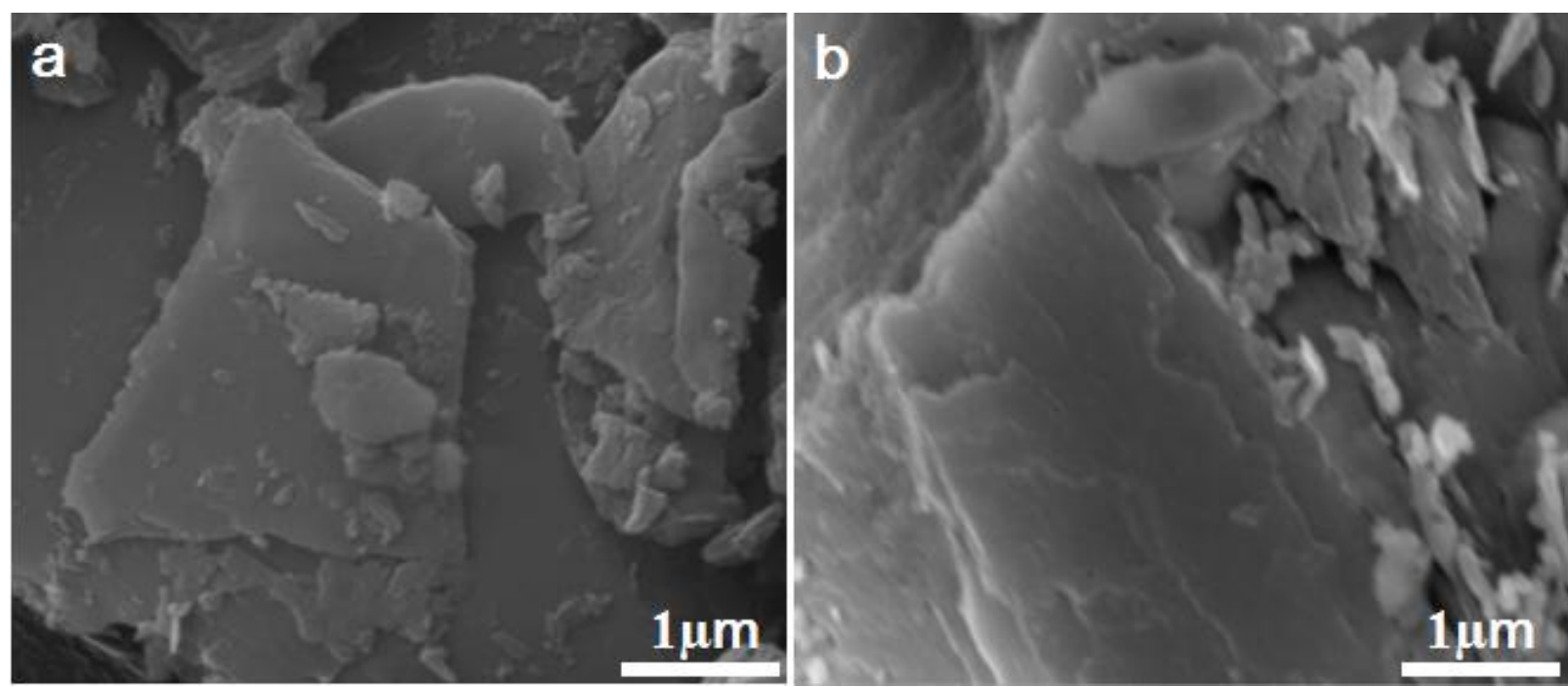

Figure S4. SEM images of as-prepared (a) $\mathrm{C}_{3} \mathrm{~N}_{4}$ and (b) B-doped $\mathrm{C}_{3} \mathrm{~N}_{4}$ nanosheets. 


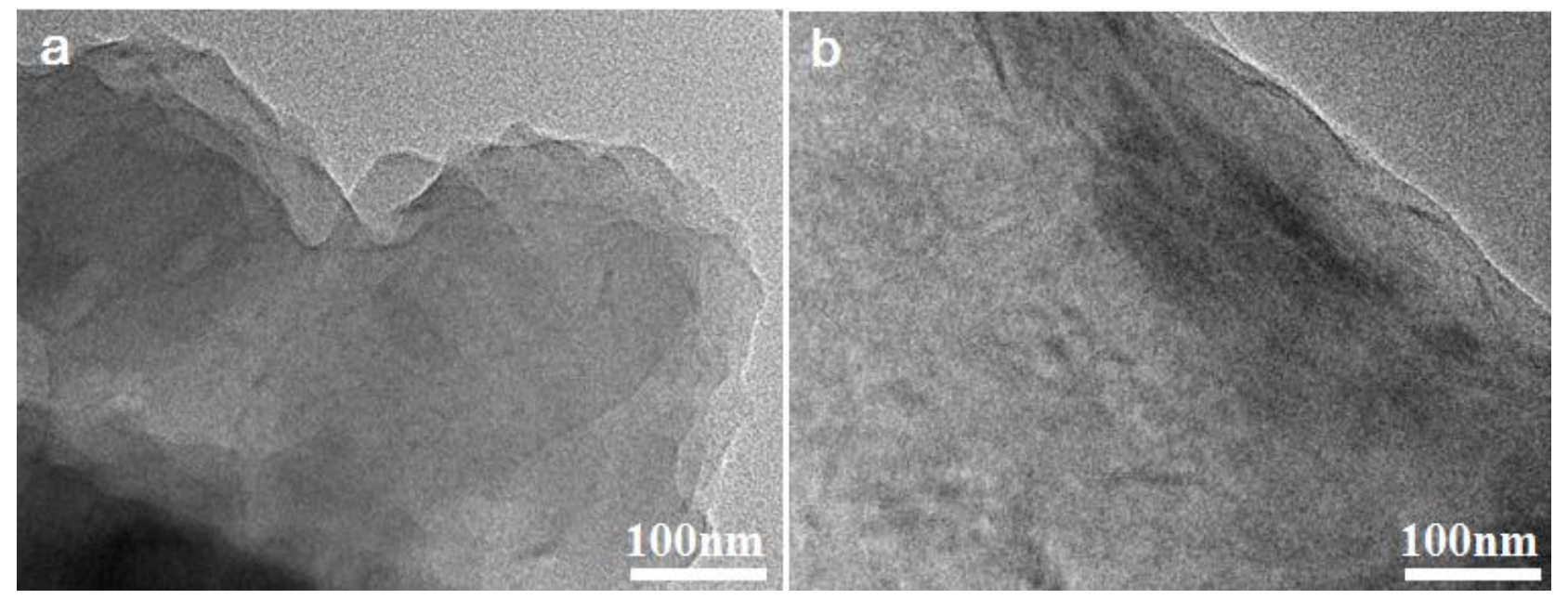

Figure S5. TEM images of as-prepared (a) $\mathrm{C}_{3} \mathrm{~N}_{4}$ and (b) B-doped $\mathrm{C}_{3} \mathrm{~N}_{4}$ nanosheets.

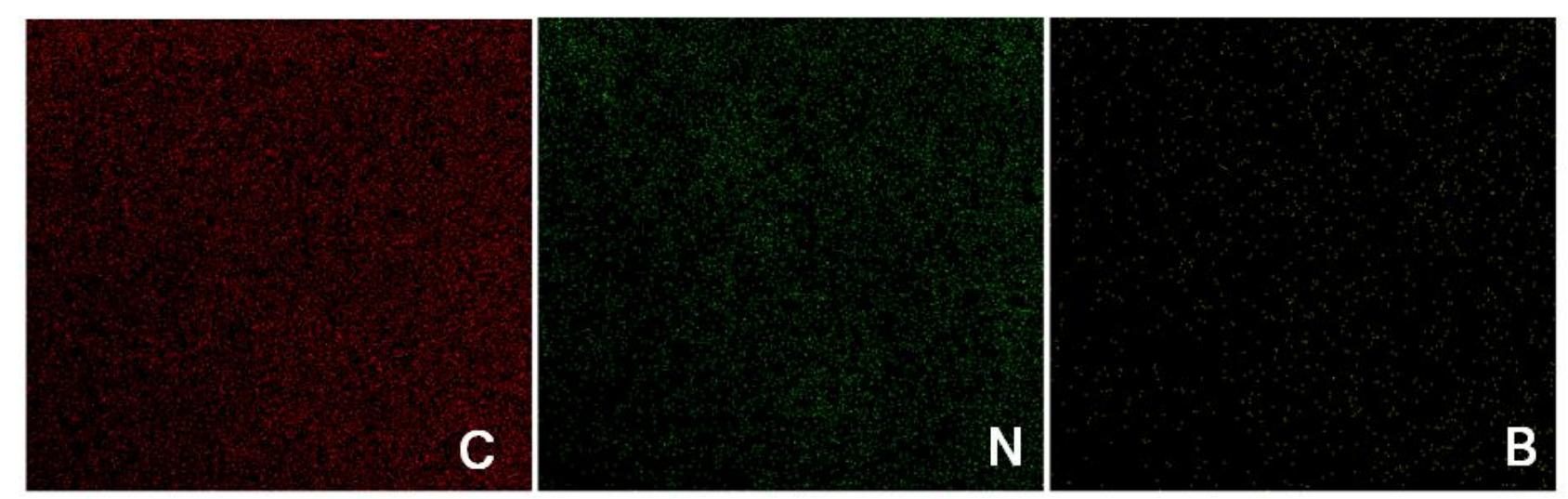

Figure S6. Elemental mapping images of $\mathrm{C}, \mathrm{N}$ and $\mathrm{B}$ of $\mathrm{B}$-doped $\mathrm{C}_{3} \mathrm{~N}_{4}$ nanosheets.

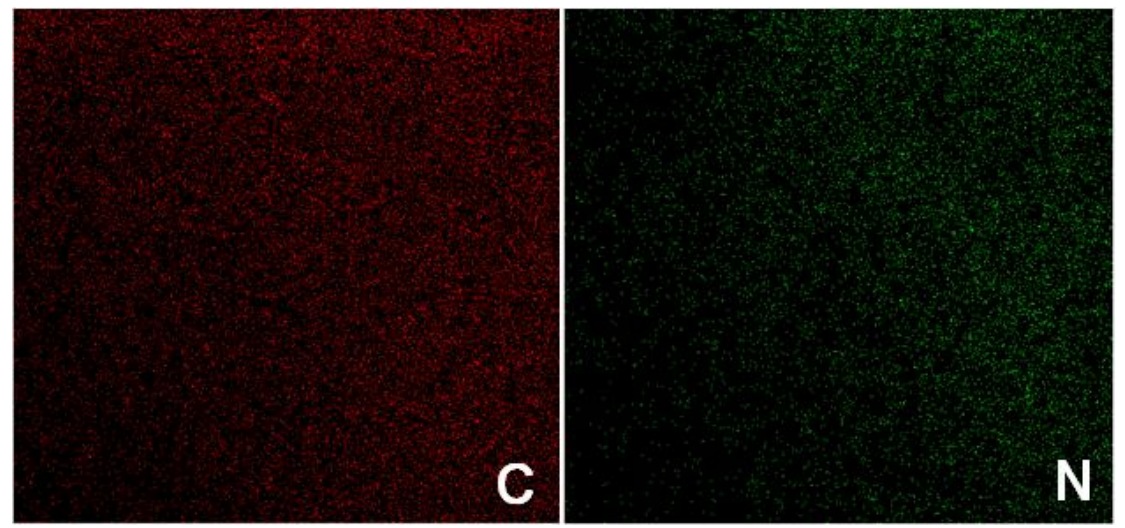

Figure S7. Elemental mapping images of $\mathrm{C}$ and $\mathrm{N}$ of $\mathrm{C}_{3} \mathrm{~N}_{4}$ nanosheets. 

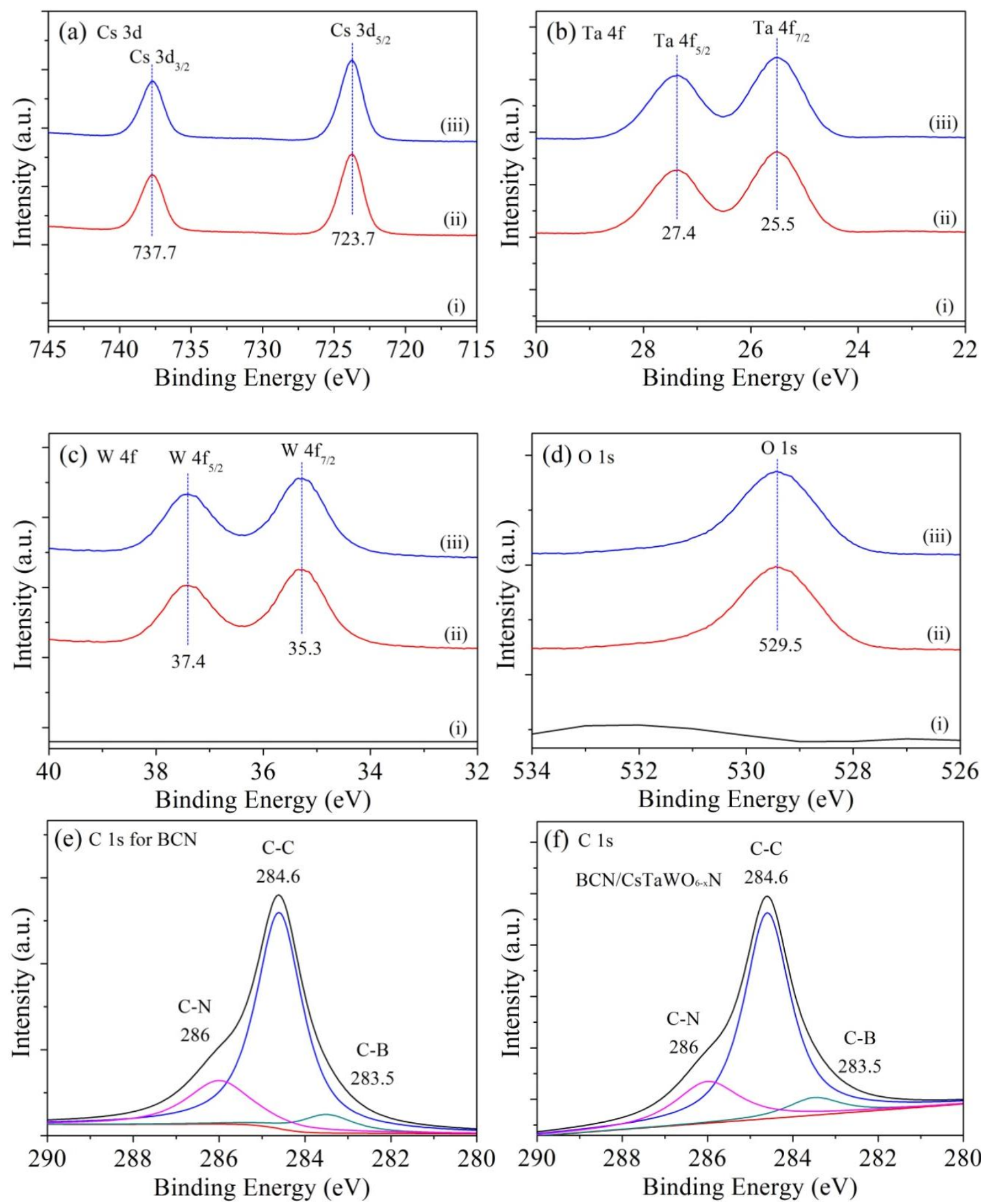

Figure S8. Cs 3d, Ta 4f, W 4f, O 1s, C 1s XPS spectra of (i) BCN, (ii) CsTaWO ${ }_{6-x} N_{x}$ and (iii) conformal $\mathrm{BCN} / \mathrm{CsTaWO}_{6-\mathrm{x}} \mathrm{N}_{\mathrm{x}}$ arrays. 

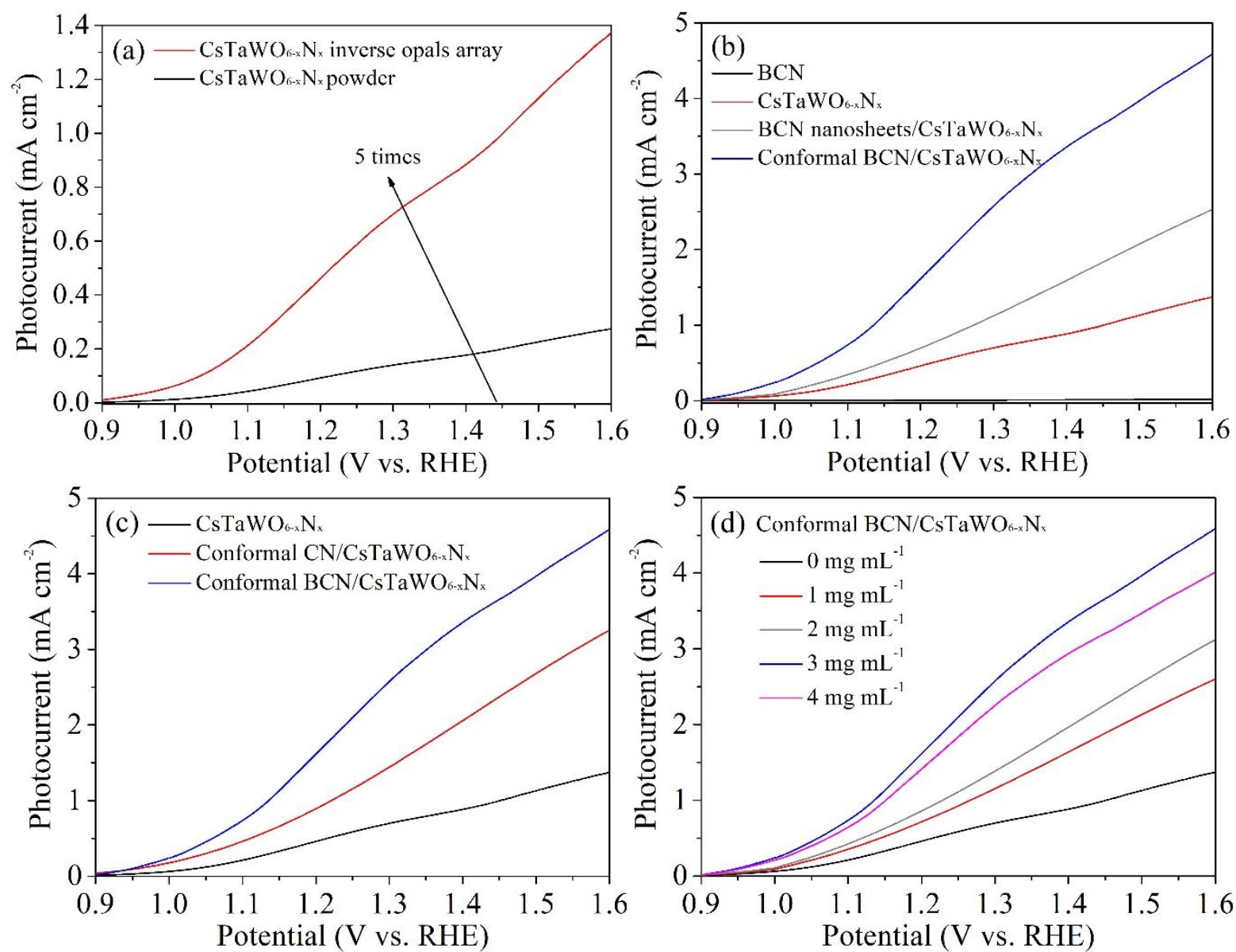

Figure S9. Photocurrent density vs. applied potential curves under AM 1.5G illumination of different photoanodes. 

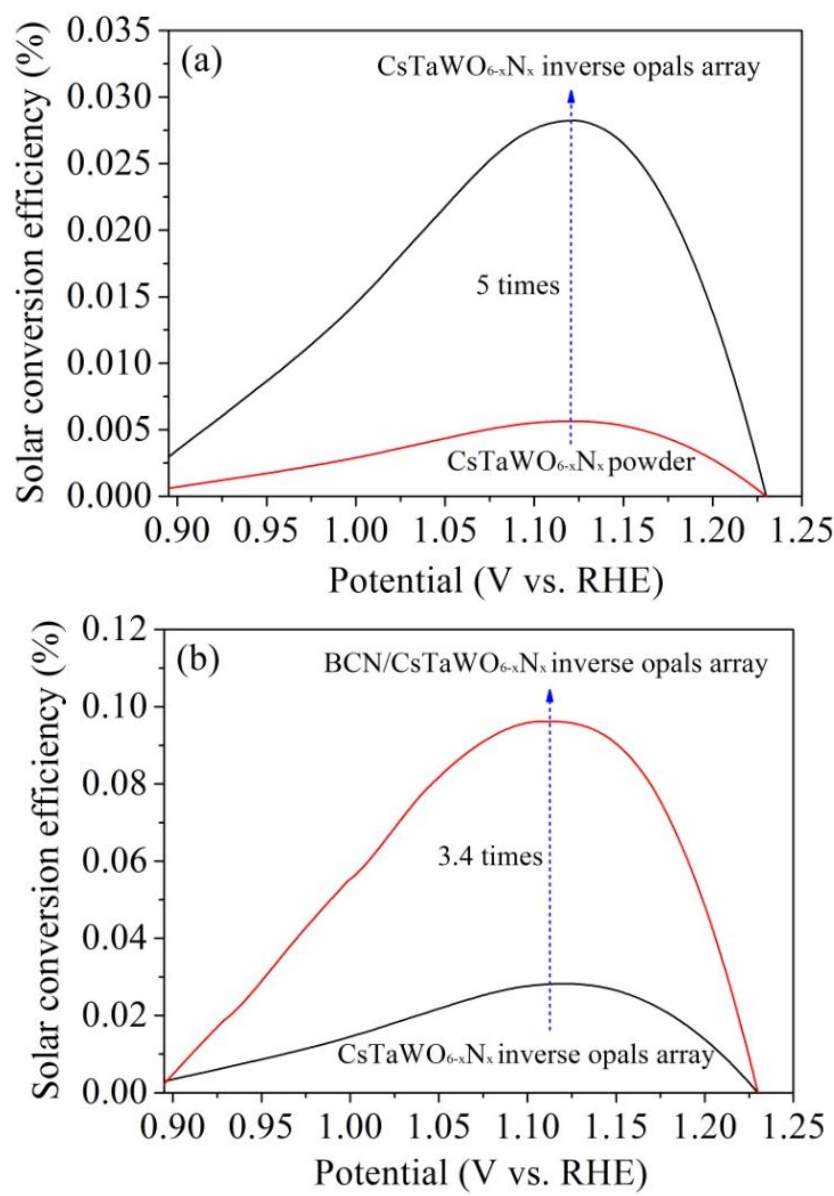

Figure S10. The solar energy conversion efficiencies of $\mathrm{CsTaWO}_{6-\mathrm{x}} \mathrm{N}_{\mathrm{x}}$ powder, $\mathrm{CsTaWO}_{6-\mathrm{x}} \mathrm{N}_{\mathrm{x}}$ inverse opals array and conformal BCN/CsTaWO $\mathrm{O}_{6-\mathrm{x}} \mathrm{N}_{\mathrm{x}}$ inverse opals array. 


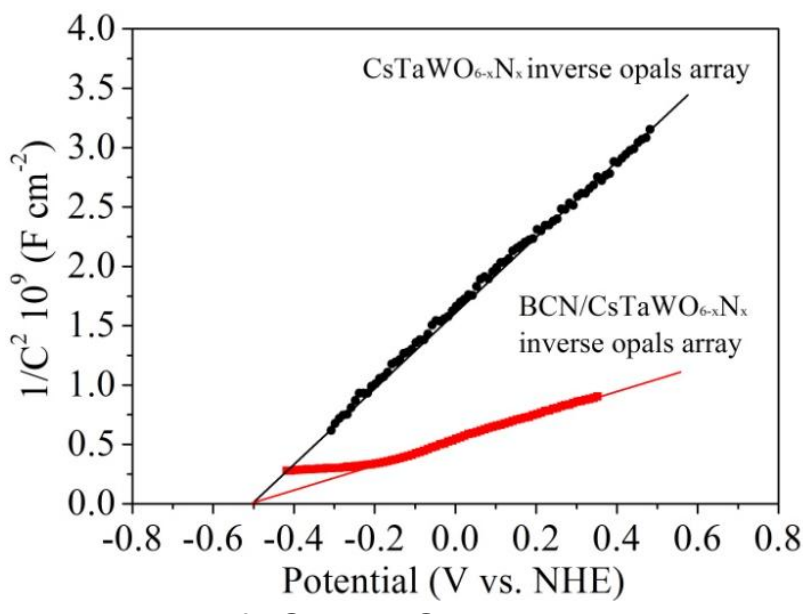

Figure S11. Mott-Schottky plots of $\mathrm{CsTaWO}_{6-x} \mathrm{~N}_{x}$ inverse opals array and conformal $\mathrm{BCN} / \mathrm{CsTaWO} \mathrm{G}_{6-\mathrm{x}} \mathrm{N}_{\mathrm{x}}$ inverse opals array measured at $1000 \mathrm{~Hz}$ in $1 \mathrm{M} \mathrm{Na}_{2} \mathrm{SO}_{4}(\mathrm{pH}=7)$.

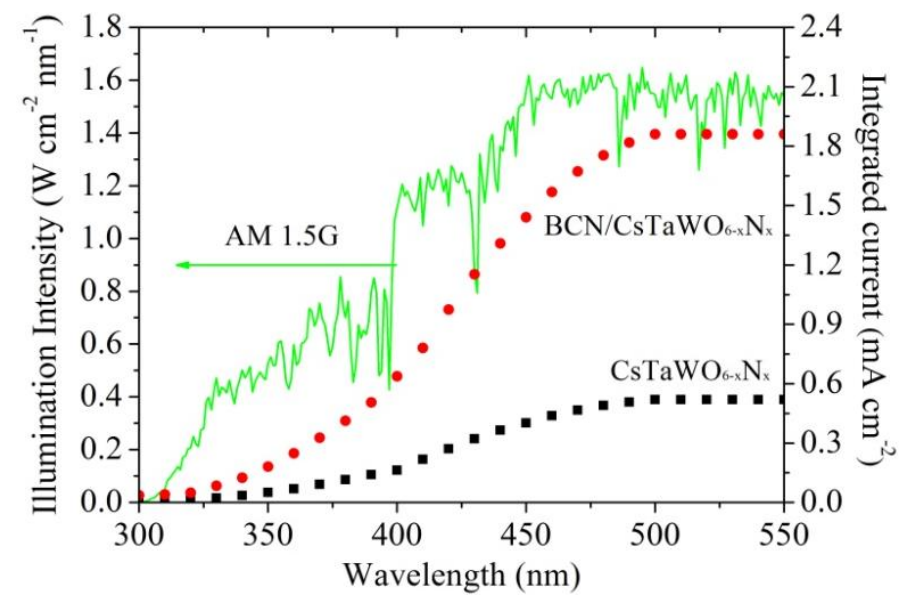

Figure S12. Solar irradiance of standard $A M 1.5 \mathrm{G}$ and calculated photocurrents by integrating IPCE over the photon flux of $A M$ 1.5G of $\mathrm{CsTaWO}_{6-x} \mathrm{~N}_{x}$ and conformal $\mathrm{BCN} / \mathrm{CsTaWO} \mathrm{G}_{6-\mathrm{x}} \mathrm{N}_{\mathrm{x}}$ photoelectrodes at the potential of $1.23 \mathrm{~V}$ vs. RHE. 

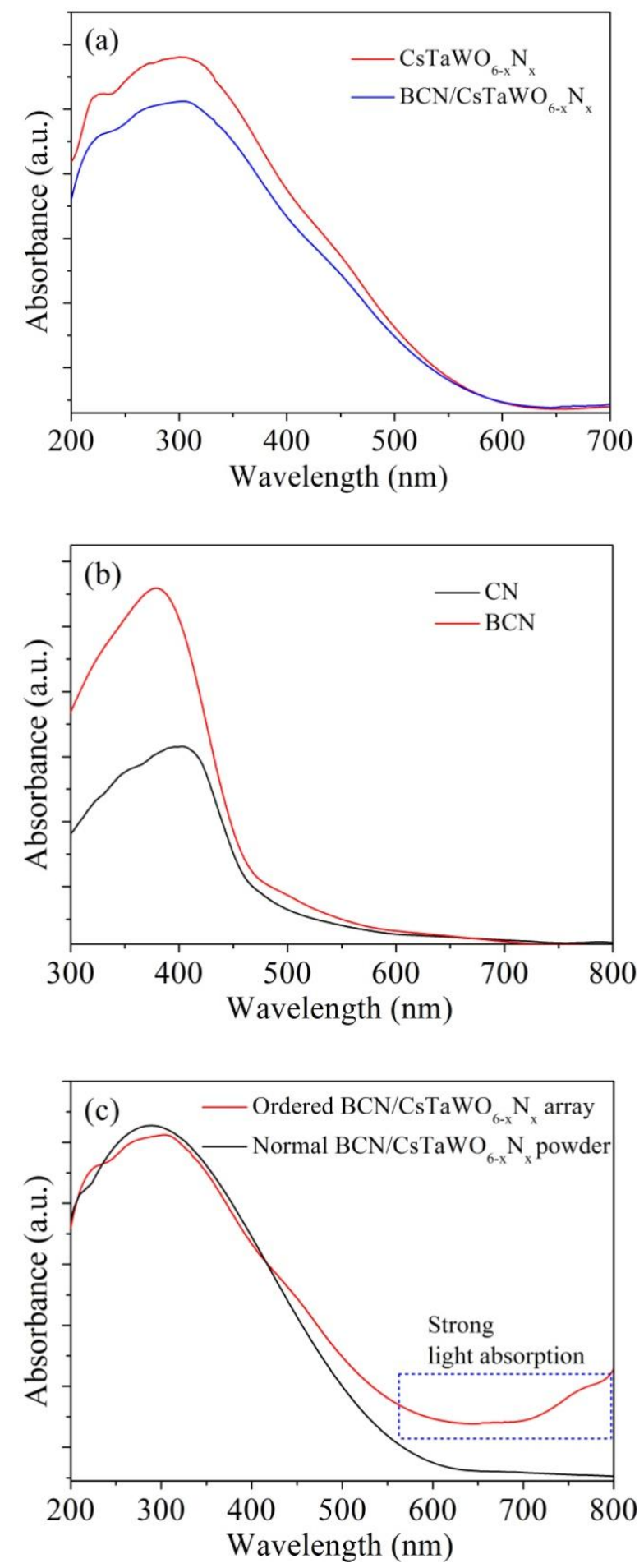

Figure S13. UV-visible absorbance spectra of (a) $\mathrm{CsTaWO}_{6-x} \mathrm{~N}_{\mathrm{x}}$ and conformal $\mathrm{BCN} / \mathrm{CsTaWO} \mathrm{O}_{6-\mathrm{x}} \mathrm{N}_{\mathrm{x}}$ arrays, (b) g- $\mathrm{C}_{3} \mathrm{~N}_{4}(\mathrm{CN})$ and boron-doped g- $\mathrm{C}_{3} \mathrm{~N}_{4}(\mathrm{BCN})$, (c) ordered $\mathrm{BCN} / \mathrm{CsTaWO}_{6-\mathrm{x}} \mathrm{N}_{\mathrm{x}}$ array in comparison of normal BCN/CsTaWO $\mathrm{O}_{6-\mathrm{x}} \mathrm{N}_{x}$ powder. 

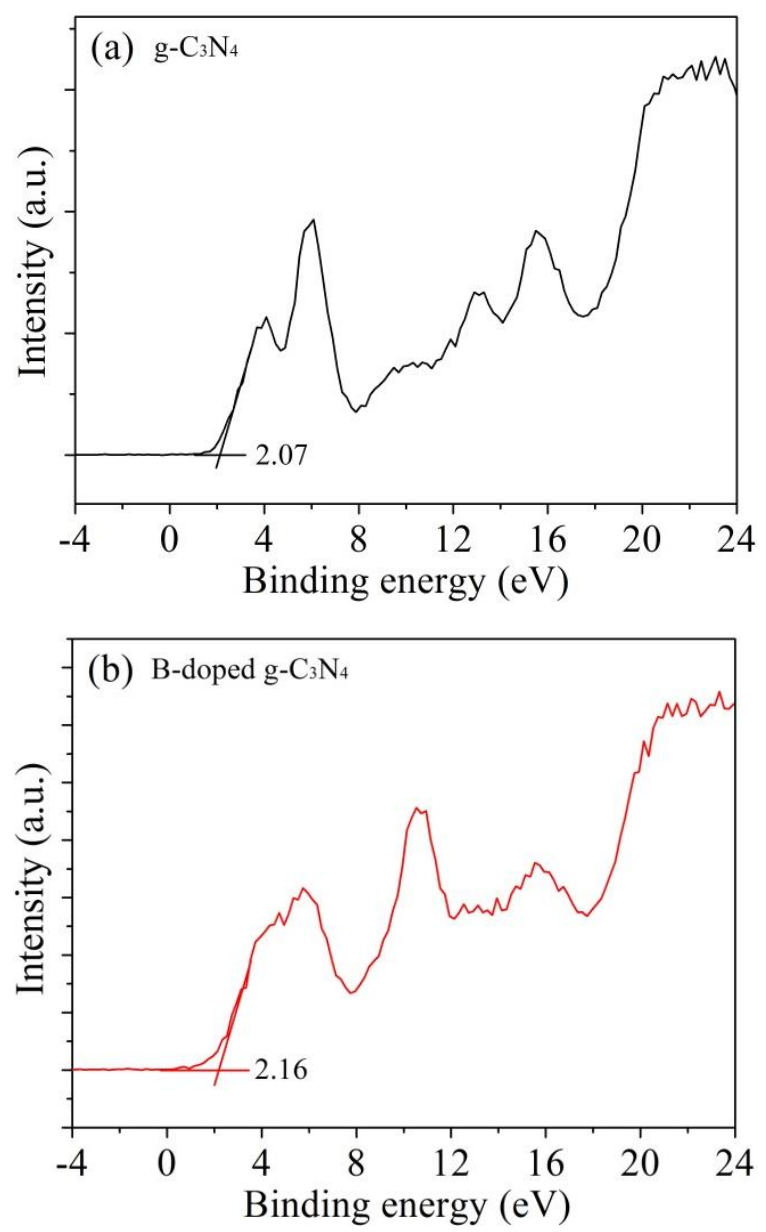

Figure S14. Valence band (VB) XPS spectra of $g-\mathrm{C}_{3} \mathrm{~N}_{4}$ and boron-doped $\mathrm{g}-\mathrm{C}_{3} \mathrm{~N}_{4}$.

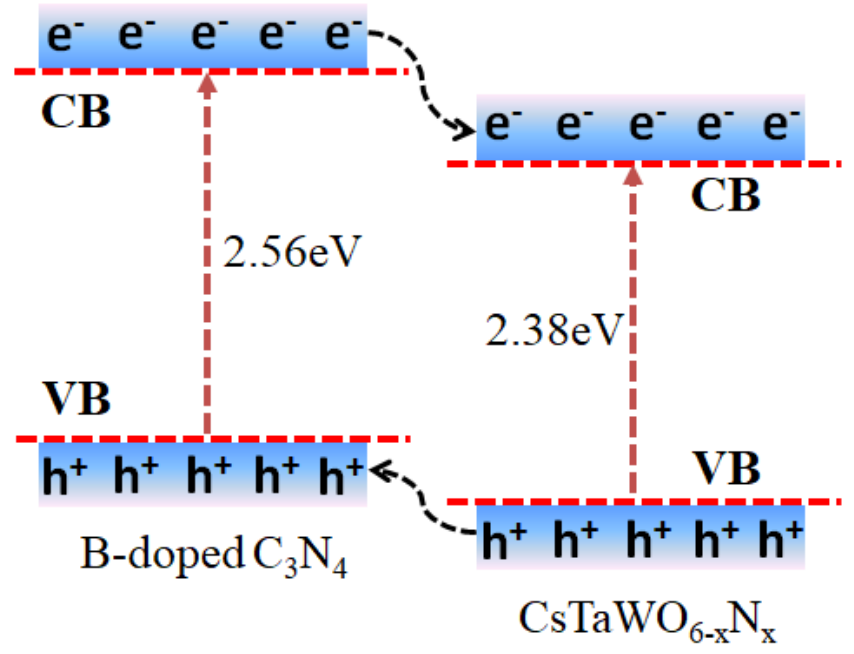

Figure S15. Band gap structures of as-prepared $\mathrm{BCN}$ and $\mathrm{CsTaWO} \mathrm{O}_{6-\mathrm{x}} \mathrm{N}_{\mathrm{x}}$ semiconductors. 

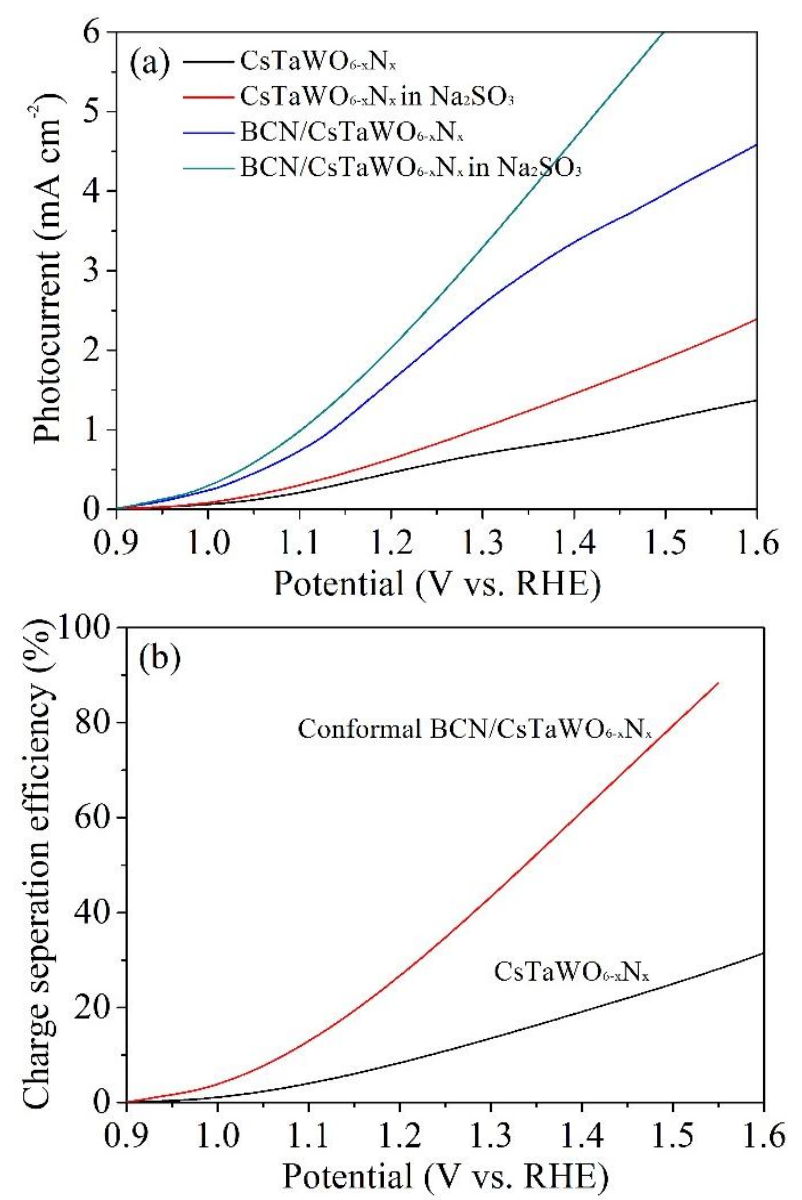

Figure S16. (a) Photocurrent density vs. applied potential curves under AM 1.5G illumination of pristine $\mathrm{CsTaWO}_{6-\mathrm{x}} \mathrm{N}_{\mathrm{x}}$ array and conformal $\mathrm{BCN} / \mathrm{CsTaWO} \mathrm{O}_{6-\mathrm{x}} \mathrm{N}_{\mathrm{x}}$ array in $\mathrm{Na}_{2} \mathrm{SO}_{3}$ solution as a hole scavenger and conventional alkaline media. (b) The calculated charge separation efficiencies of $\mathrm{CsTaWO}_{6-\mathrm{x}} \mathrm{N}_{\mathrm{x}}$ and conformal BCN/CsTaWO $\mathrm{O}_{6-\mathrm{x}} \mathrm{N}_{\mathrm{x}}$ arrays.

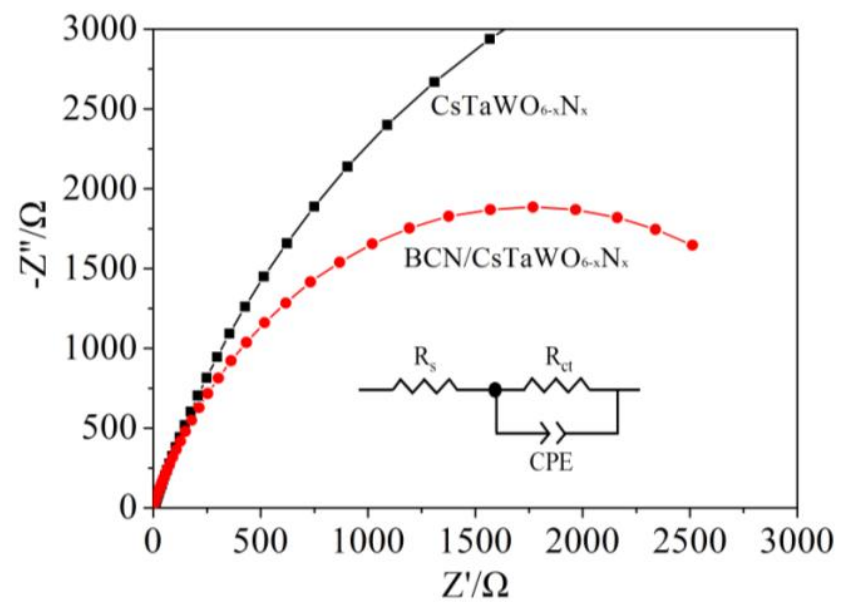

Figure S17. Electrochemical impedance spectra (EIS) of $\mathrm{CsTaWO}_{6-\mathrm{x}} \mathrm{N}_{\mathrm{x}}$ and conformal $\mathrm{BCN} / \mathrm{CsTaWO} \mathrm{O}_{6-\mathrm{x}} \mathrm{N}_{\mathrm{x}}$ arrays. 

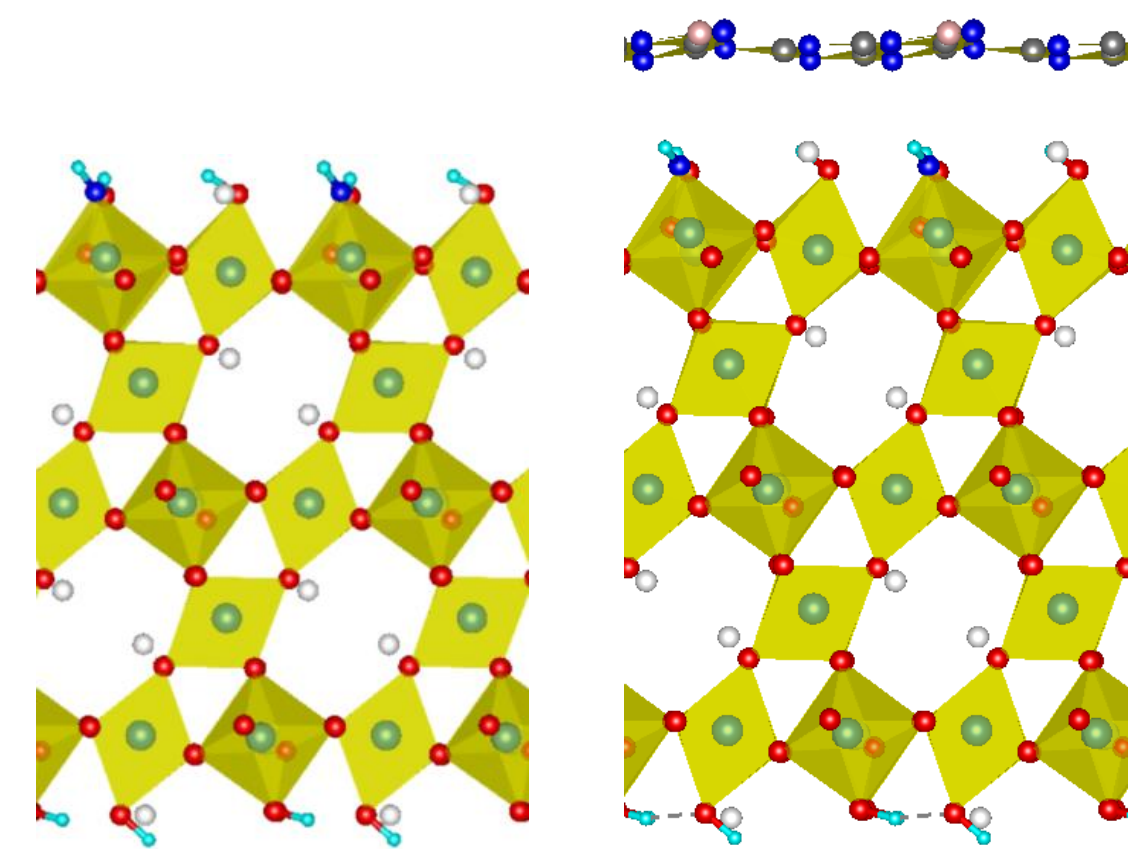

Figure S18. Atomic structure models of CsTaWO ${ }_{6-x} \mathrm{~N}_{x}$ and $\mathrm{BCN} / \mathrm{CsTaWO} \mathrm{O}_{6-x} \mathrm{~N}_{x}$. 
Table S1. PEC water splitting performance of $B C N / C s T a W O_{6-x} N_{x}$ photoelectrode in comparison of various photoelectrodes.

\begin{tabular}{|c|c|c|c|}
\hline Materials & Electrolyte & Photocurrent & References \\
\hline $\begin{array}{l}\mathrm{BCN} / \mathrm{CsTaWO}_{6-\mathrm{x}} \mathrm{N}_{\mathrm{x}} \\
\text { array }\end{array}$ & $1.0 \mathrm{M} \mathrm{KOH}$ & $\begin{array}{l}4.59 \mathrm{~mA} \mathrm{~cm}^{-2} \text { at } 1.6 \mathrm{~V} \\
\text { vs. RHE }\end{array}$ & This work \\
\hline $\begin{array}{l}\mathrm{BCN} / \mathrm{CsTaWO}_{6-\mathrm{x}} \mathrm{N}_{\mathrm{x}} \\
\text { array }\end{array}$ & $1.0 \mathrm{M} \mathrm{KOH}$ & $\begin{array}{l}1.9 \mathrm{~mA} \mathrm{~cm}^{-2} \text { at } 1.23 \mathrm{~V} \\
\text { vs. } \mathrm{RHE}\end{array}$ & This work \\
\hline $\mathrm{BaTaO}_{2} \mathrm{~N}$ & $\begin{array}{c}0.5 \mathrm{M} \mathrm{K}_{2} \mathrm{HPO}_{4} \\
(\mathrm{pH}=13)\end{array}$ & $\begin{array}{l}0.75 \mathrm{~mA} \mathrm{~cm}^{-2} \text { at } 1.23 \mathrm{~V} \\
\text { vs. RHE }\end{array}$ & $\begin{array}{l}\text { Adv. Funct. Mater. } \\
\text { 2016, 26, 7156. }\end{array}$ \\
\hline $\mathrm{SrTaO}_{2} \mathrm{~N}$ & $1.0 \mathrm{M} \mathrm{NaOH}$ & $\begin{array}{l}1.1 \mathrm{~mA} \mathrm{~cm}^{-2} \text { at } 1.23 \mathrm{~V} \\
\text { vs. } \mathrm{RHE}\end{array}$ & $\begin{array}{l}\text { Adv. Funct. Mater. } \\
2016,26,7156 \text {. }\end{array}$ \\
\hline LaTiO $_{x} \mathrm{~N}_{\mathrm{y}}$ & $0.5 \mathrm{M} \mathrm{NaOH}$ & $\begin{array}{l}<0.3 \mathrm{~mA} \mathrm{~cm}^{-2} \text { at } 1.5 \mathrm{~V} \\
\text { vs. } \mathrm{RHE}\end{array}$ & $\begin{array}{l}\text { Adv. Funct. Mater. } \\
2017,27,1605690 .\end{array}$ \\
\hline $\mathrm{BaNbO}_{2} \mathrm{~N}$ & $\begin{array}{c}0.5 \mathrm{M} \mathrm{KBi} \\
(\mathrm{pH}=13)\end{array}$ & $\begin{array}{l}0.85 \mathrm{~mA} \mathrm{~cm}^{-2} \text { at } 1.23 \mathrm{~V} \\
\text { vs. } \mathrm{RHE}\end{array}$ & $\begin{array}{c}\text { Chem. Mater. 2016, } \\
28,6869 .\end{array}$ \\
\hline $\mathrm{SrNbO}_{2} \mathrm{~N}$ & $\begin{array}{l}0.5 \mathrm{M} \mathrm{KBi} \\
(\mathrm{pH}=13)\end{array}$ & $\begin{array}{l}0.53 \mathrm{~mA} \mathrm{~cm}^{-2} \text { at } 1.23 \mathrm{~V} \\
\text { vs. } \mathrm{RHE}\end{array}$ & $\begin{array}{c}\text { Chem. Mater. 2016, } \\
28,6869 .\end{array}$ \\
\hline $\begin{array}{l}\text { Boron-incorporated } \\
\mathrm{Ta}_{3} \mathrm{~N}_{5}\end{array}$ & $\begin{array}{c}0.1 \mathrm{M} \mathrm{Na}_{2} \mathrm{SO}_{4} \\
5 \mathrm{M} \mathrm{NaOH} \\
(\mathrm{pH}=13)\end{array}$ & $\begin{array}{l}0.95 \mathrm{~mA} \mathrm{~cm}^{-2} \text { at } 1.23 \mathrm{~V} \\
\text { vs. } \mathrm{RHE}\end{array}$ & $\begin{array}{c}\text { ACS Appl. Mater. } \\
\text { Interfaces 2017, 9, 42, } \\
36715\end{array}$ \\
\hline $\mathrm{CaFe}_{2} \mathrm{O}_{4} / \mathrm{TaON}$ & $\begin{array}{c}0.5 \mathrm{M} \mathrm{NaOH} \\
(\mathrm{pH}=13.7)\end{array}$ & $\begin{array}{l}0.7 \mathrm{~mA} \mathrm{~cm}^{-2} \text { at } 1.23 \mathrm{~V} \\
\text { vs. } \mathrm{RHE}\end{array}$ & $\begin{array}{l}\text { J. Am. Chem. Soc. } \\
2013,135,5375 .\end{array}$ \\
\hline $\mathrm{LaTaON}_{2}$ & $1.0 \mathrm{M} \mathrm{NaOH}$ & $\begin{array}{l}0.8 \mathrm{~mA} \mathrm{~cm}^{-2} \text { at } 1.6 \mathrm{~V} \\
\text { vs. } \mathrm{RHE}\end{array}$ & $\begin{array}{l}\text { Appl. Catal. B 2018, } \\
\text { 226.111. }\end{array}$ \\
\hline $\mathrm{TiO}_{2}$ array & $1.0 \mathrm{M} \mathrm{NaOH}$ & $\begin{array}{l}1.24 \mathrm{~mA} \mathrm{~cm}^{-2} \text { at } 1.23 \mathrm{~V} \\
\text { vs. } \mathrm{RHE}\end{array}$ & Small, 2016, 12, 1469. \\
\hline $\mathrm{ZnO} / \mathrm{ZnS} / \mathrm{g}-\mathrm{C}_{3} \mathrm{~N}_{4}$ & $0.5 \mathrm{M} \mathrm{Na}_{2} \mathrm{SO}_{4}$ & $\begin{array}{l}0.66 \mathrm{~mA} \mathrm{~cm}^{-2} \text { at } 1.23 \mathrm{~V} \\
\text { vs. } \mathrm{RHE}\end{array}$ & $\begin{array}{l}\text { Adv. Mater. Interfaces } \\
\text { 2017. 1700681. }\end{array}$ \\
\hline $\ln _{2} \mathrm{O}_{3-x} / \mathrm{In}_{2} \mathrm{~S}_{3}$ & $1.0 \mathrm{M} \mathrm{KOH}$ & $\begin{array}{l}1.28 \mathrm{~mA} \mathrm{~cm}^{-2} \text { at } 1.23 \mathrm{~V} \\
\text { vs. } \mathrm{RHE}\end{array}$ & $\begin{array}{l}\text { Adv. Energy Mater. } \\
2018,8,1701114 \text {. }\end{array}$ \\
\hline $\mathrm{TiO}_{2} / \mathrm{CdS} / \mathrm{CoP}$ & $\begin{array}{c}0.1 \mathrm{M} \\
\text { phosphate }\end{array}$ & $\begin{array}{l}<1.2 \mathrm{~mA} \mathrm{~cm}^{-2} \text { at } 1.23 \mathrm{~V} \\
\text { vs. } \mathrm{RHE}\end{array}$ & $\begin{array}{l}\text { Adv. Funct. Mater. } \\
2015,25,5706 .\end{array}$ \\
\hline $\mathrm{SrTiO}_{3}$ & $0.5 \mathrm{M} \mathrm{NaOH}$ & $\begin{array}{l}<0.5 \mathrm{~mA} \mathrm{~cm}^{-2} \text { at } 1.5 \mathrm{~V} \\
\mathrm{Ag} / \mathrm{AgCl}\end{array}$ & $\begin{array}{c}\text { Nano Lett. } 2016,16, \\
7338 .\end{array}$ \\
\hline $\mathrm{Fe}_{2} \mathrm{O}_{3}$ & $0.1 \mathrm{M} \mathrm{NaOH}$ & $\begin{array}{l}<0.3 \mathrm{~mA} \mathrm{~cm}^{-2} \text { at } 1.23 \mathrm{~V} \\
\text { vs. } \mathrm{RHE}\end{array}$ & $\begin{array}{l}\text { Adv. Funct. Mater. } \\
2016,26,4435 .\end{array}$ \\
\hline $\mathrm{ZnFe}_{2} \mathrm{O}_{4}$ & $0.1 \mathrm{M} \mathrm{NaOH}$ & $\begin{array}{l}<0.3 \mathrm{~mA} \mathrm{~cm}^{-2} \text { at } 1.23 \mathrm{~V} \\
\text { vs. } \mathrm{RHE}\end{array}$ & $\begin{array}{l}\text { Adv. Funct. Mater. } \\
2016,26,4435 .\end{array}$ \\
\hline $\mathrm{TiO}_{2}$ & $1.0 \mathrm{M} \mathrm{KOH}$ & $\begin{array}{l}0.73 \mathrm{~mA} \mathrm{~cm}^{-2} \text { at } 1.5 \mathrm{~V} \\
\text { vs. } \mathrm{RHE}\end{array}$ & $\begin{array}{l}\text { ACS Nano } 2012,6, \\
5060 .\end{array}$ \\
\hline $\mathrm{BiVO}_{4}$ & $1.0 \mathrm{M} \mathrm{NaOH}$ & $\begin{array}{l}0.66 \mathrm{~mA} \mathrm{~cm}^{-2} \text { at } 1.23 \mathrm{~V} \\
\text { vs. } \mathrm{RHE}\end{array}$ & $\begin{array}{c}\text { Adv. Mater. 2017, 29, } \\
1605308 .\end{array}$ \\
\hline $\mathrm{AZO} / \mathrm{TiO}_{2} / \mathrm{Au}$ & $0.1 \mathrm{M} \mathrm{Na}_{2} \mathrm{SO}_{4}$ & $\begin{array}{l}1.1 \mathrm{~mA} \mathrm{~cm}^{-2} \text { at } 1.23 \mathrm{~V} \\
\text { vs. } \mathrm{RHE}\end{array}$ & $\begin{array}{l}\text { Adv. Energy Mater. } \\
2016,6,1501496 .\end{array}$ \\
\hline
\end{tabular}




\section{References.}

1. Zhou, M. et al. Ordered macroporous $\mathrm{BiVO}_{4}$ architectures with controllable dual porosity for efficient solar water splitting. Angew. Chem. Int. Ed. 52, 8579-8583 (2013).

2. Zhou, M. et al. Photoelectrodes based upon $\mathrm{Mo}: \mathrm{BiVO}_{4}$ inverse opals for photoelectrochemical water splitting. ACS Nano, 8, 7088-7098 (2014).

3. Kresse, G. \& Hafner, J. Ab initio molecular dynamics for liquid metals. Phys. Rev. B 47, 558 (1993).

4. Perdew, J. P., Burke, K., Ernzerhof, M. Generalized gradient approximation made simple. Phys. Rev. Lett. 77, 3865 (1996).

5. Kresse, G., Joubert, D. From ultrasoft pseudopotentials to the projector augmentedwave method. Phys. Rev. B 59, 1758 (1999).

6. Grimme, S., Antony, J., Ehrlich, S., Krieg, H. Effect of the damping function in dispersion corrected density functional theory. J. Chem. Phys. 132, 154104 (2010). 